This is the peer-reviewed version of the article:

Mitic, V., Lazovic, G., Milosevic, D., Lu, C.-A., Manojlovic, J., Tsay, S.-C.,

Kruchinin, S., Vlahovic, B., 2020. Brownian motion and fractal nature.

Mod. Phys. Lett. B 34, 2040061. https://doi.org/10.1142/S0217984920400618

\title{
Brownian motion and fractal nature
}

\author{
Vojislav Mitic ${ }^{*, \dagger, \S}$, Goran Lazovic ${ }^{\dagger, \uparrow}$ and Dusan Milosevic ${ }^{\ddagger}, \|$ \\ * Institute Technical Sciences of SASA University, Belgrade, Serbia \\ ${ }^{\dagger}$ Faculty of Electronic Engineering, University Nis, Nis, Serbia \\ $\ddagger$ Faculty of Mechanical Engineering, University of Belgrade, Belgrade, Serbia \\ $\S$ vmitic.d2480@gmail.com \\ 『glazovic@mas.bg.ac.rs \\ "dusan.milosevic@elfak.ni.ac.rs \\ Chun-An Lu \\ Industrial Technology Research Institute, Taiwan \\ chunan.lu@gmail.com \\ Jelena Manojlovic** \\ Faculty of Mechanical Engineering, University of Nis, Nis, Serbia \\ jmanojlo@gmail.com \\ Shwu-Chen Tsay \\ National Tsing Hua University, Taiwan \\ tsay.susan@gmail.com \\ Sergey Kruchinin \\ Bogolyubov Institute for Theoretical Physics, \\ National Ukrainian Academy of Sciences, Ukraina \\ sergeikruchinin@yahoo.com \\ Branislav Vlahovic \\ North Carolina Central University, Durham NC, USA \\ vlahovic@nccu.edu
}

The main goal of our research is to find the connection between micro particles and microorganisms motion in the Nature, considered as Brownian's Motion within the fractal's nature. For ceramics and generally material science it is important to clarify the particles motion and other phenomena, especially for grains and pores. Our idea is to establish control over the relation order-disorder on particle motion and their collision

** Corresponding author. 
effects by Brownian motion phenomena in the frame of fractal nature matter. We performed some experiments and got interesting results based on microorganism motion initiated by different outer energetic impulses. This is practically the idea of biomimetic correlation between particles and microorganisms Worlds, what is very original and leads towards biunivocal different phenomena's understanding. Another idea is to establish some controlling effects for electro ceramic particle motion in chemical-materials sciences consolidation by some phenomena in the nature. These important research directions open new frontiers with very specific reflections for future of microelectronics materials.

Keywords: Microparticles; microorganisms; Brownian motion; fractals.

\section{General Appearance}

Microelectronics phenomena in different submicrostructures, high-level parameter integrations, and packing, are very important in many aspects of fundamental research. On one hand it is important to fulfill many goals on the way to miniaturization, but for such scientific questions there are many open problems that are very creative for advanced investigations. Anyhow, scientific perspectives in classic semiconductor technologies are not with an open vision to give new fundamental results. There are strong technological limitations to make thinner and thinner layers for microelectronics demands. Our research tries to open new frontiers in the fields of electronics parameter integrations, instead of different microdevices technological integration (Fig. 1). But this scientific and research problem is not easy to understand, because of many open questions and not enough investigated problems and appropriate answers. One of these aspects is a much deeper and more precise control of particle motion and their trajectories phenomena changes.

Therefore, we have developed research results in the field of Brownian particle motion. Also, here we have applied fractal nature in Brownian motion chaotic structures. In that way, we have practically developed mathematical-physics methods to control chaotic motion and transform to control structures on the way to real particles order. From these points of view, we are coming to the point to explain and understand some processes in the nature of material sciences, especially ceramic structures. For these problems we have opened frontiers to use some ideas of similarities in the motion of particles from phenomena in the live systems, and to compare with particle motion in inorganic world. All of these are specific ideas of biomimetic similarities. These Brownian particle motion effects have a great importance for advanced miniaturization and packaging, presented on Fig. 1.

Some of our experimental results with submicroorganisms of size between $0.5 \mu \mathrm{m}$ and $3 \mu \mathrm{m}$ are inspiration for Brownian motion fractal nature analysis, which could be applicable for particles such as electrons. For better understanding of these processes, we provide some necessary tools for further research.

\subsection{Short intro into Brownian motion and fractal analysis}

Brownian motion is a stochastic process $\{B(t), t \geq 0\}$ with the following properties: 


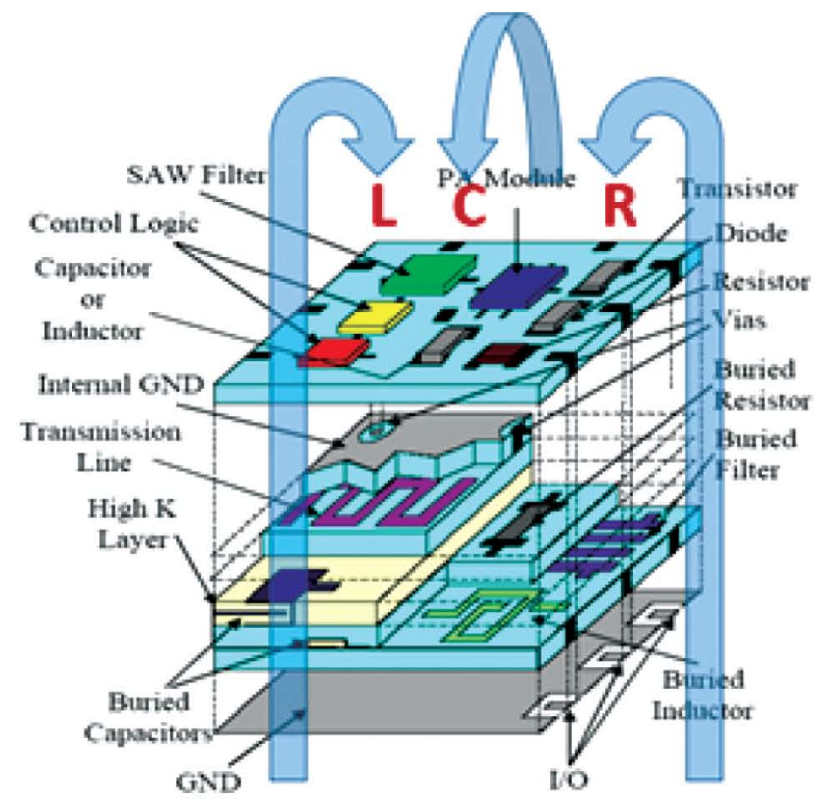

Fig. 1. (Color online) Low temperature co-fired ceramic (LTCC) module based on integrations microdevices and Resistance, Capacitance, Inductance (RCL) parameters. ${ }^{a}$

- $B(0)=0$;

- for any $0 \leq t_{0}<t_{1}<\cdots<t_{n}$, random variables $B\left(t_{\mathrm{k}}\right)-B\left(t_{\mathrm{k}-1}\right) ; 1 \leq k \leq n$ are independent;

- for $0 \leq s<t, B(t)-B(s)$ is normally distributed with mean $\mu(t-s)$ and variance $\sigma^{2}(t-s)$, where $\mu$ and $\sigma \neq 0$ are real numbers.

This process will be called a $(\mu, \sigma)$ Brownian motion with drift $\mu$ and variance $\sigma^{2}$. It can be supposed $\mu=0, \sigma=1$ and we get:

$$
B(t)-B(0)=B(t): N(0, t),
$$

i.e. $N(0, t)$ is normally distributed with mean 0 and variance $t$.

On the other hand, Brownian motion can be obtained as limit of rescaled simple random walks. Let $\xi 1, \xi 2, \ldots$ be a sequence of independent, identically distributed random variables with mean 0 and variance 1 . For each $n \geq 1$ define continuous-time stochastic process $\left\{B_{n}(t), t \geq 0\right\}$ by:

$$
B_{n}(t)=\frac{1}{\sqrt{n}} \sum_{1 \leq j \leq\lfloor n t\rfloor} \xi_{j} .
$$

a Thanks to cooperation with Professor J. J. Jean from National Tsing Hua University, Taiwan. 


\section{2D Fractal Interpolation by IFS}

In this paper we will consider Fractal Interpolation Function (FIF) based on the theory of Iterated Function Systems (IFS). ${ }^{1-6}$ First let us involve some definitions:

Definition 2.1. ${ }^{5}$ A metric space is a pair $(M, d)$, where $M$ is a non-empty set and $d: M \rightarrow \mathbb{R}$ is a real valued function called a metric on $M$, with the following properties:

(i) Positive definite: for all $x, y \in M, d(x, y) \geq 0$

(ii) Symmetric: for all $\boldsymbol{x}, y \in M, d(x, y)=d(y, x)$

(iii) Triangle inequality for all $x, y, z \in M, d(x, y) \leq d(x, z)+d(z, y)$.

Definition $2.2 .{ }^{5}$ Let a pair $(M, d)$ be a metric space and let " $a$ " be the family of all closed subsets of $M$. For $r>0$ and $A \in a$, let $V_{r}(A)=\{m: d(m, a)<r\}$, and definite for all $A, B \in a$, Hausdorff metric

$$
d^{\prime}(A, B)=\inf \left\{r: A \subset V_{r}(B) \wedge B \subset V_{r}(A)\right\} .
$$

Let $(M, d)$ be a compact metric space and $H$ be set of all nonempty closed subsets of $M$. Then $(H, d)$ is a compact metric space with the Hausdorff metric. A set of contractions $w_{i}: M \rightarrow M$ for all $i=1,2, \ldots, n$ is called, according to $M$. Barnsley, ${ }^{2}$ an IFS.

Further, for any subset $A$ of $H$, let us define function $W: H \rightarrow H$ by

$$
W(A)=\bigcup_{i} w_{i}(A) .
$$

This function is contraction on $H$. According to the Banach contraction principle, there is a unique set $G \in H$, such that $W(G)=G$. This set $G$ is an attractor for the IFS. According to Barnsley ${ }^{1}$ the IFS always admits at least one attractor.

An IFS is hyperbolic if for all $x, y \in M$ holds inequality

$$
d\left(w_{i}(x), w_{i}(y)\right) \leq s d(x, y),
$$

where $s \in[0,1)$.

For the hyperbolic IFS, $W$ is a contraction mapping, that is, for all $A, B \in H$ holds,

$$
h(W(A), W(B)) \leq s h(x, y)
$$

In that case, $W$ has a unique attractor.

In the sequel, we will consider the case when $M=\mathbb{R}^{2}$. First, let us define a set of interpolation data:

Definition 2.3. ${ }^{2}$ A collection of data of the form

$$
\left\{\left(x_{i}, F_{i}\right) \in \mathbb{R}^{2}, i=0,1,2, \ldots, n\right\},
$$

where $n$ is a positive integer, $F_{i}=F\left(x_{i}\right)$ and $x_{0}<x_{1}<x_{2}<\cdots<x_{n}$ is a set of interpolation data. 
The corresponding interpolation function is continuous function $f:\left[x_{0}, x_{n}\right] \rightarrow \mathbb{R}$, such that $f\left(x_{i}\right)=F_{i}$, for all $i=0,1, \ldots, n$. We shall say that interpolation function $f$ interpolates interpolation data and that graph of the function passes through points $\left\{\left(x_{i}, F_{i}\right)\right\}$.

\section{Experimental Part}

In our experiments, we performed $\mathrm{BaTiO}_{3}$ sample preparation with solvent procedure. We started with pure $\mathrm{BaTiO}_{3}$ nano structured powders with different additives as illustrated in Fig. 2.

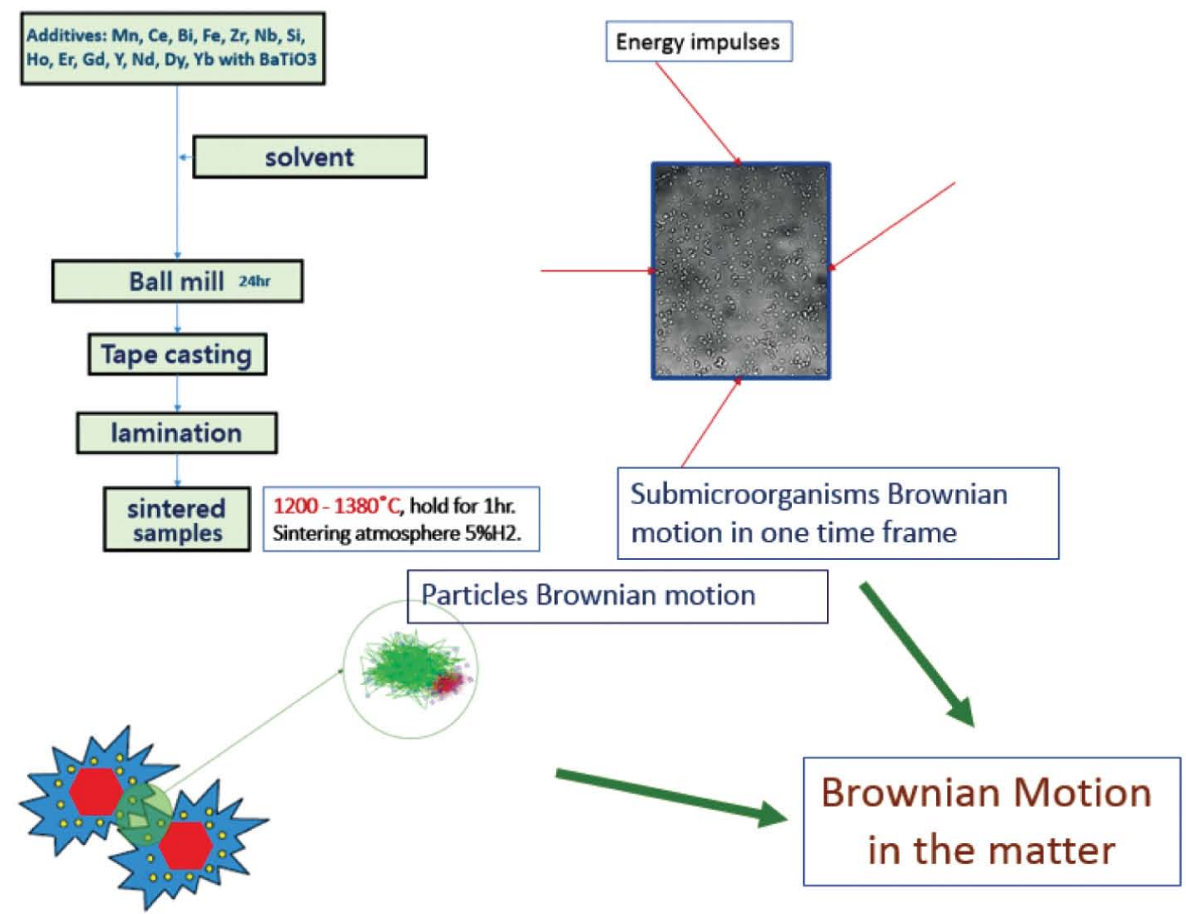

Fig. 2. (Color online) The experimental procedure diagram, which generalizes Brownian motion as characteristic of the matter.

All of these have been prepared by specific and protected ITRI procedure (one group of experiments, ${ }^{b}$ and second group with living organisms at National Tsing Hua University). The consolidation process was according to algorithm (Fig. 2). The size of samples was $3 \mathrm{~cm}$ in diameter, and had an average thickness of 0.75 microns. The sintering has been held for $1 \mathrm{hr}$ at atmosphere $5 \% \mathrm{H}_{2} / \mathrm{N}_{2}$. The consolidation parameters and testing conditions are presented in Table 1.

${ }^{\mathrm{b}}$ Industrial Technology Research Institute, Taiwan. 
Table 1. Consolidation parameters and testing conditions.

\begin{tabular}{|c|c|c|}
\hline & Different additives (Fig. 2) & $\begin{array}{c}\text { Element-block polymer different } \\
\text { additives-doping }\end{array}$ \\
\hline $\mathrm{BaTiO}_{3} 50 \mathrm{~nm}$ & $\begin{array}{c}\text { Green tape } \\
\text { Sintering temp. } 1200-1350^{\circ} \mathrm{C}\end{array}$ & $\begin{array}{c}\text { Green tape } \\
\text { Sintering temp. } 1200-1350^{\circ} \mathrm{C}\end{array}$ \\
\hline $\mathrm{BaTiO}_{3} 200 \mathrm{~nm}$ & Green tape & Green tape \\
& Sintering temp. $1200-1350^{\circ} \mathrm{C}$ & Sintering temp. $1200-1350^{\circ} \mathrm{C}$ \\
\hline
\end{tabular}

In Fig. 2, below the samples consolidation diagram, we extracted one capple of ceramics grains, where we notify the particle (electrons) intergranular fluctuations, which are basically characterized by Brownian motion.

Simultaneously, with experimental procedure based on ceramics powders and samples with different additives, we have developed the other part of experiment, related to submicroorganisms within the liquid in one chamber (right side, Fig. 2.).

The goal of this unified experimental presentation is developing and demonstration of the asymptotic approaching Brownian motion, within solid state in ceramics and live organisms in the liquid at the end as one in the nature.

\subsection{The Brownian motion in submicroorganisms experiments}

We have developed some experimental procedures based on energy impulses influence in one liquid medium with some submicroorganisms. We have got some results in order to demonstrate changes in the motions and trajectories of some submicroorganisms. These results are in very short time intervals and exposed to microstructures, as illustrated in Fig. 3.
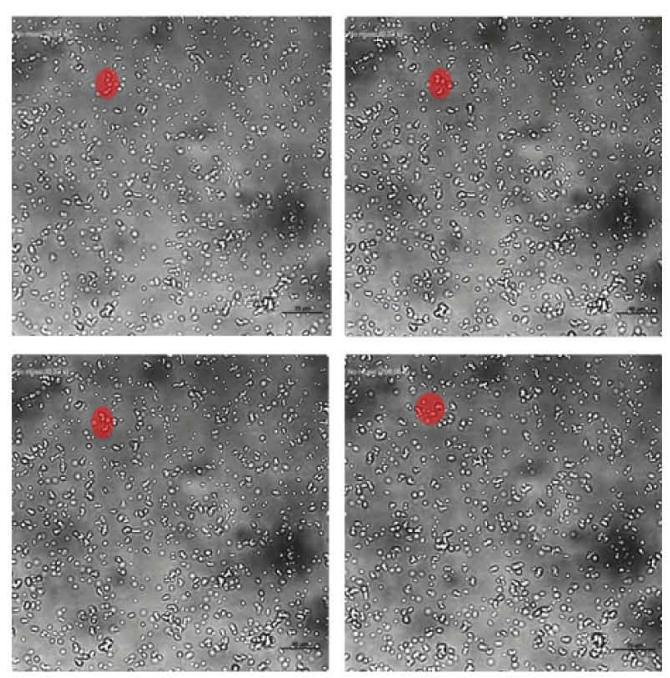

Fig. 3. (Color online) Observed submicroorganisms. 

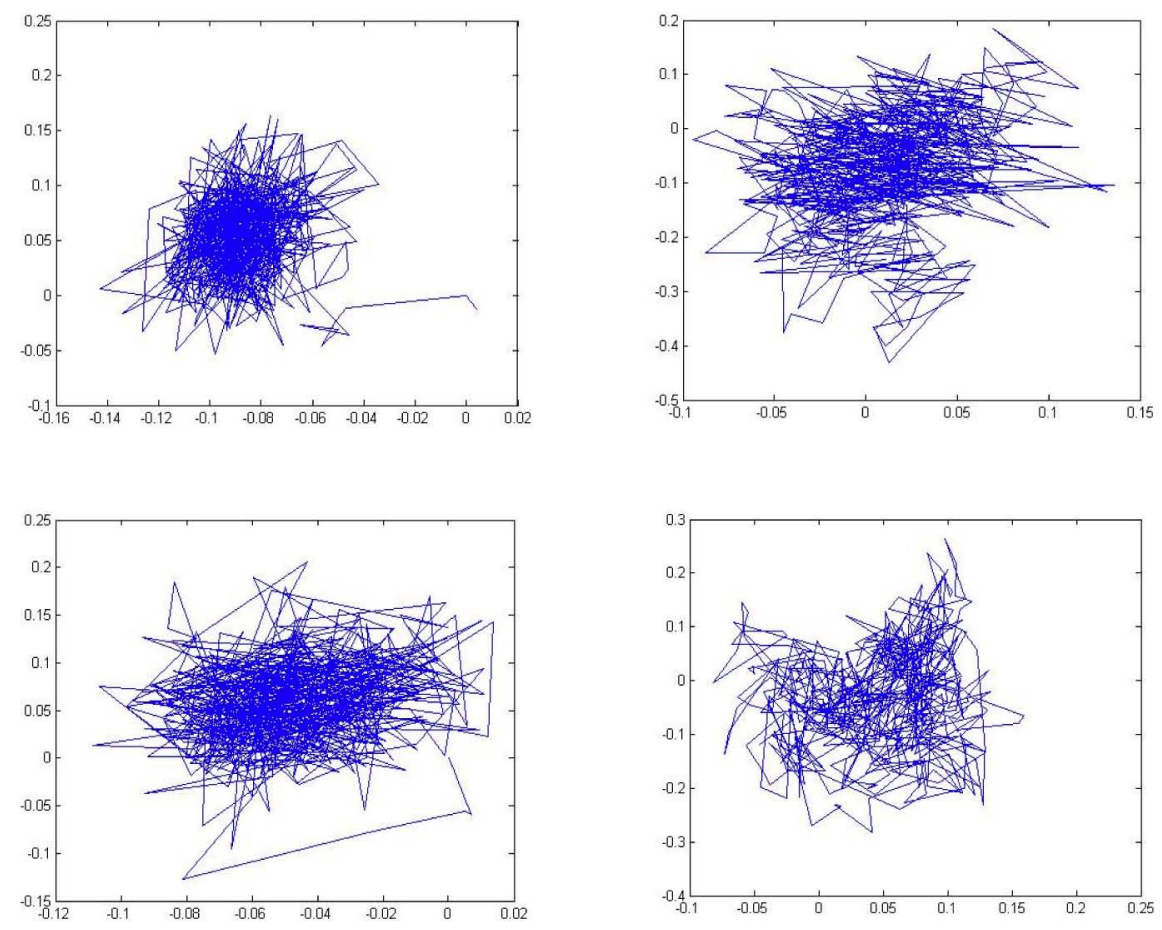

Fig. 4. (Color online) 2D trajectories.

Because of the very short intervals in which we analyzed the submicroorganism Brownian motion, there are 1/60 s time interval in which 600 figures were extracted, which represent phase cross section in this dynamic motion process. We selected four images to express the microorganism motion, as a result of influence of different energy impulses on experimental area.

Practically, we have established a relation between the microparticles over grain boundaries, like electrons, which jump from one grain to another characterized by Brownian motion from one side, and submicroorganisms in specific Brownian motion from another side. We can observe the motion of microorganisms in some of the figures in this paper. Based on these figures we have analyzed Brownian motion, as illustrated in Fig. 4.

For submicroorganism kinetics we selected four and disposed their complex trajectories.

Furthermore, we have provided results regarding the fractal nature analysis applied in the previous Brownian motion diagrams.

A random segment of the first submicroorganism trajectory is selected. Further, selected segment is considered as interpolation data, so fractal interpolation function could be constructed. Fig. 5, shows fractal interpolation functions for various scaling factor. 


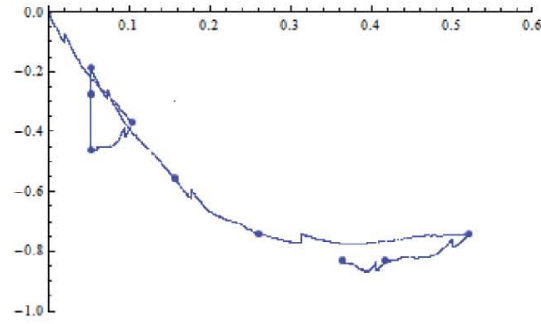

Scaling factor $=0.1$

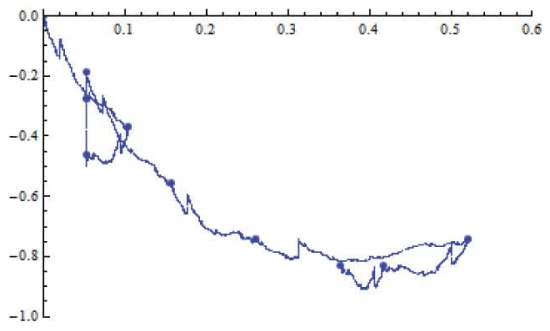

Scaling factor $=0.2$

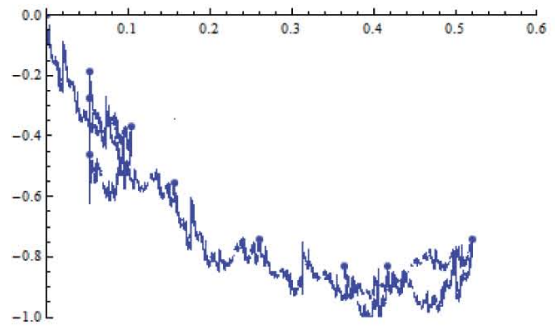

Scaling factor $=0.4$

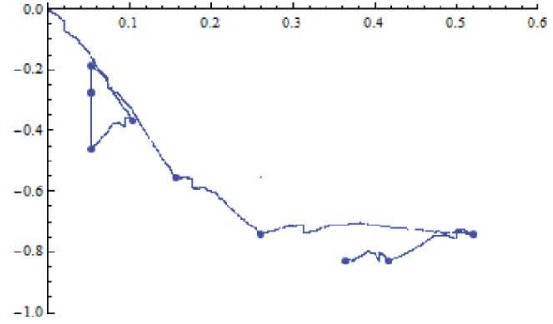

Scaling factor $=-0.1$

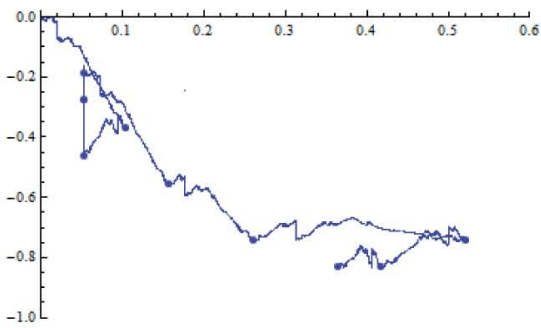

Scaling factor $=-0.2$

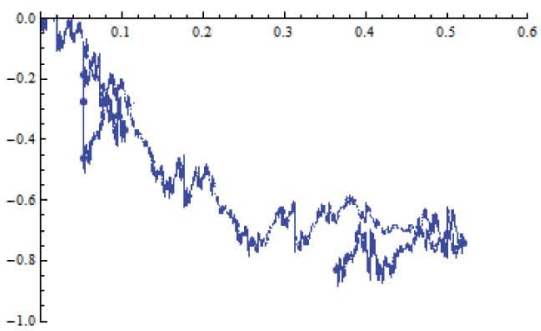

Scaling factor $=-0.4$

Fig. 5. (Color online) Fractal interpolated 2D trajectories.

\section{Results and Discussion}

These results are for Brownian motion and fractal analysis in 2D. Also, furthermore we have designed 3D diagrams, which correspond to 2D Brownian motion results and we can observe them below.

These 3D results and diagrams definitely confirmed the real images of submicroorganism motions in space. These structures are also very complex, but open the idea to apply the results, which we used for fractalization of electron trajectories. ${ }^{6,7}$

By these experimental and theoretical results, we verified that all of these point sets correspond to real submicrorganism Brownian motion, which could be represented in general form. 

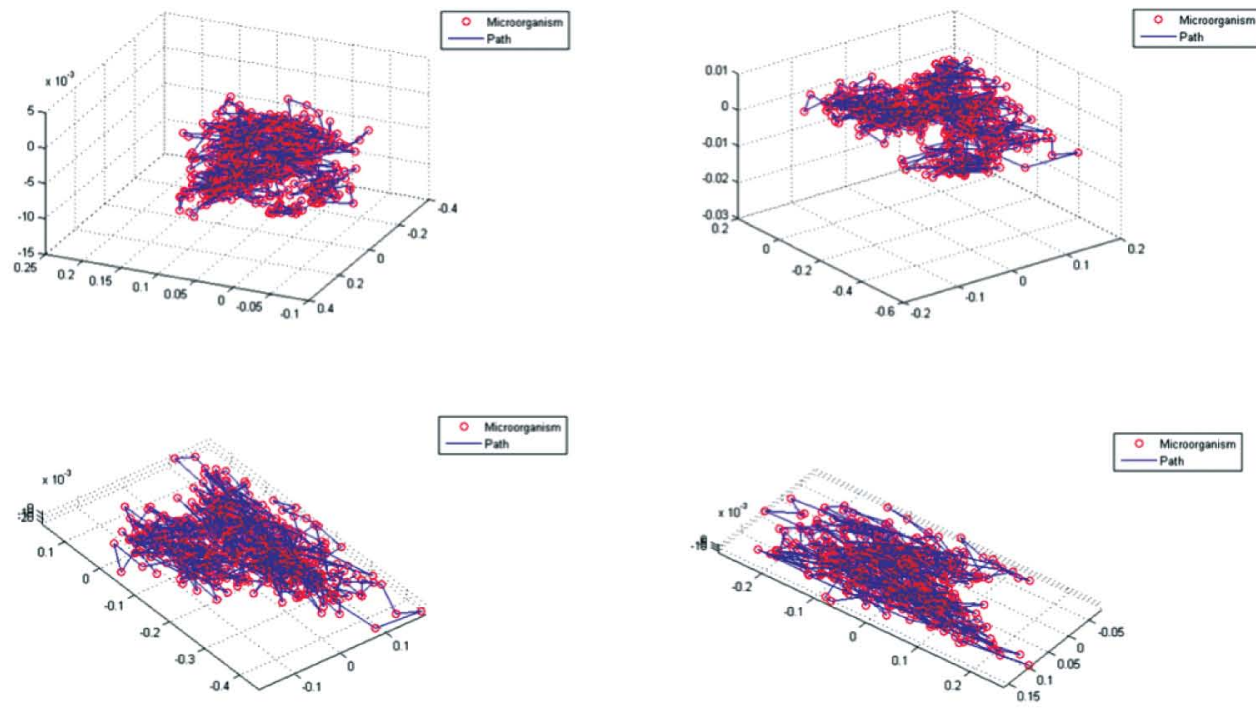

Fig. 6. (Color online) 3D trajectories.
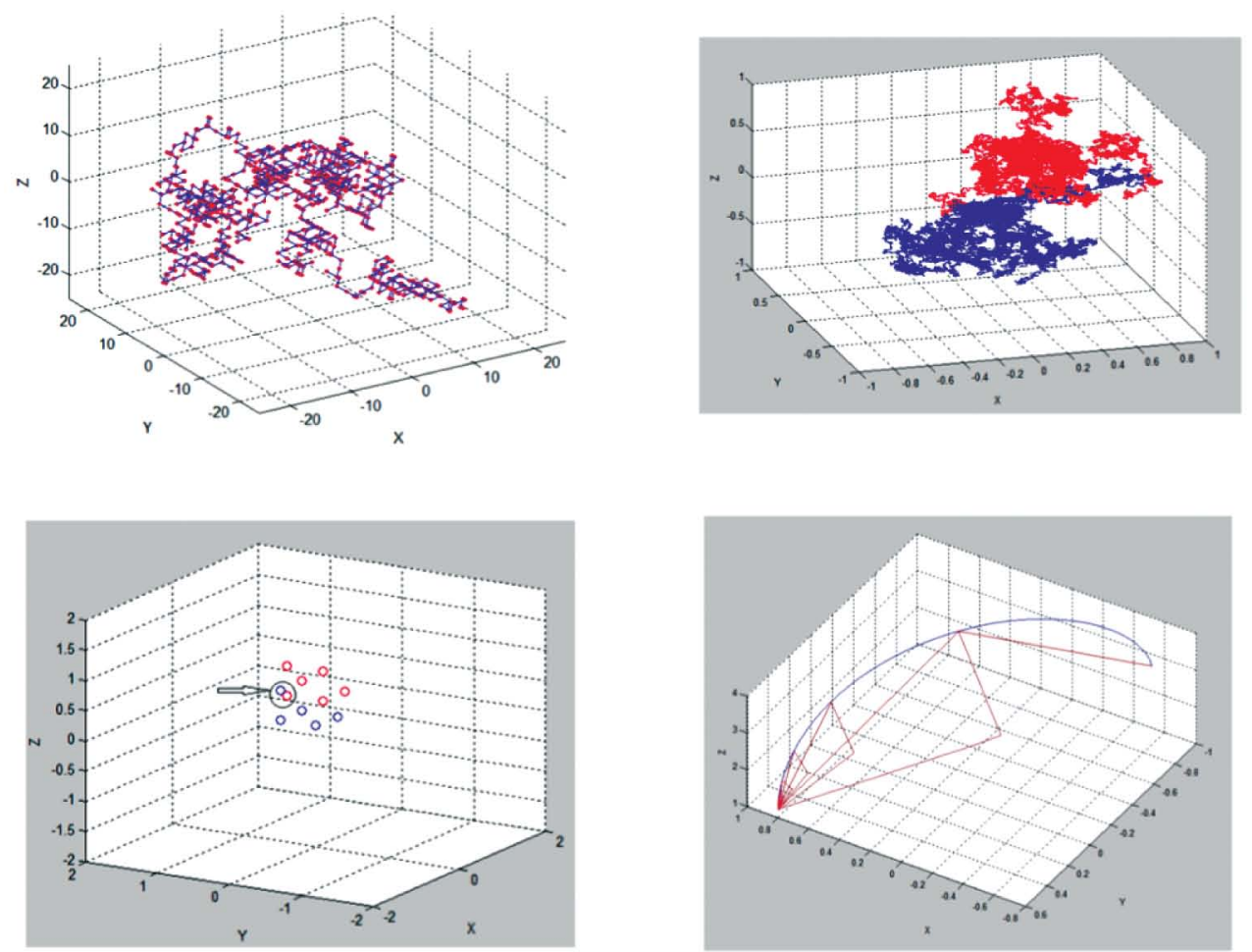

Fig. 7. (Color online) Presentation of electrons jumping from one intergranular boundary to another, with colision effects and fractal particles trajectory. 
On the other hand, some of our results define the original tool for particle (electrons) kinetics between two grains in ceramics samples. A very important point in all of this is the trajectories fractalization possibility.

So, we definitely can transform and control different particle trajectories that are very complex, like submicroorganism motion. These practically open one, like biomimetic approach, to meet in one point of the analysis of Brownian motion and fractal nature of microorganisms and microparticles. In this research, we will not consider the differences in volumes and masses of submicroorganisms and microparticles, but only the similarities in their motion, kinetic dynamics as ending and beginning of the live world and states nature within matter in general.

When we consider the electron kinetics, we understand that in this process we always have the electron clouds of one side of grain boundary in "preparation" to jump these grain boundaries and also another grain boundary of a different grain. Definitely, in every other grain we have particles that are already in the area next to that grain boundary. So, it is a very real scenario, where we will get the collision between the electron cloudsparticles. This very complex dynamic process among the particles, produce trajectories of the particles after collision and under different local electrostatic fields, which are very chaotic curvatures. From that point of view, it is very important to develop some methods to control these types of kinetics (Fig. 3).

If we would like to have a better control in understanding the particle motion, what causes different related electronic, dielectric, and ferroelectric parameters. Our approach considers the high-slevel microelectronics integrations based on different parameters integrations and complex biunivocal influences. Our scientific approach in this contact point between electrons and submicroorganisms opens additional view on generalized motion and kinetics in nature, from the points of view of particles from both the live and inanimate worlds. We can apply in general the idea of fractalization of electron trajectories also on very complex Brownian motion trajectories.

\section{Outlook}

Regarding the intention to connect micromotions in fractalized Brownian motion of particles like electrons and the submicroorganism world with specific Brownian motion, we will consider future experiments with will close and compare this phenomena as one asymptotic process among small particles in inorganic structures and submicroorganisms in nature. These two models are practically coming from the same matter phenomena. So, our future experiments are toward the direction that is practically closing the changes and motions in the inorganic inanimate nature with alive nature phenomena, as just only one complex process.

\section{Conclusion}

In our paper we open a quite new enlightenment in similar processes between particle motion in matter and submicroorganisms motion characterized by 
fractal nature as just motions in matter like only one exists. We successfully demonstrated different experiments and methods to recognize real microparticle motion in inanimate nanoceramics structures. On the other hand, based on experiments with live microorganisms we recognized the fractal nature in Brownian motion of such case. So definitely in one asymptotically predicted procedure, we have opened one frontier to understand that in both cases we have one real Brownian motion fractal nature case.

\section{References}

1. M. F. Barnsley, Constr. Approx. 2 (1986) 303.

2. M. F. Barnsley, Fractals Everywhere (Academic Press, San Diego, 1993).

3. J. Hutchinson, Indiana U. Math. J. 30 (1981) 713.

4. N. A. Secelean, Far East J. Dyn. Syst. 3 (2001) 149.

5. S. P. Kruchinin, V. F. Klepikov, V. E. Novikov and D. S. Kruchinin, Mater. Sci. 23(4) (2005) 1009.

6. S. Kruchinin, V. Novikov and V. Klepikov, Metrol. Meas. Syst. 15 (2008) 281.

7. A. Helemskii, Lecture and Exercises on Functional Analysis (American Mathematical Society, RI, 2006).

8. V. V. Mitić, V. Paunović, G. Lazović, Lj. Kocić and B. Vlahović, Ferroelectrics 536 (2018) 60.

9. Z. B. Vosika, V. V. Mitić, G. Lazović, V. Paunović and Lj. Kocić Ferroelectrics 531 (2018) 38 . 